\title{
Série de Casos: Gliomas de Alto Grau do Hospital de Clínicas de Passo Fundo - HCPF - RS
}

Julia Pastorello ${ }^{1,2}$, Emanuela Lando ${ }^{2,3 *}$, Camila dos Santos do Amaral ${ }^{1,2}$, Cristiane Pagnussat Cechetti ${ }^{1,2}$, Giovana Zerwes Vacaro ${ }^{1,2}$, Nicoli Taiana Henn ${ }^{1,2}$, Marina Ractz Bueno ${ }^{1,2}$, Douglas Carvalho Cogo ${ }^{1,2}$, Robson Rottenfusser ${ }^{4}$, Paulo Moacir Mesquita Filho ${ }^{5}$

\footnotetext{
${ }^{1}$ Departamento de Oncologia Clínica, Hospital de Clínicas de Passo Fundo - HCPF, Passo Fundo, Rio Grande do Sul/RS, Brasil.

${ }^{2}$ Liga Acadêmica de Oncologia e Hematologia do Hospital de Clínicas de Passo Fundo - LAONC- HCPF, Hospital de Clínicas de Passo Fundo - HCPF, Passo Fundo, Rio Grande do Sul/RS, Brasil.

${ }^{3}$ Acadêmica de Medicina, Faculdade Meridional - IMED, Passo Fundo, Rio Grande do Sul/RS, Brasil.

${ }^{4}$ Médico Radiologista, Hospital de Clínicas de Passo Fundo - HCPF, Passo Fundo, Rio Grande do Sul/RS, Brasil.

${ }_{5}^{5}$ Médico Neurocirurgião, Hospital de Clínicas de Passo Fundo - HCPF, Passo Fundo, Rio Grande do Sul/RS, Brasil.
}

*Autor Correspondente: Emanuela Lando. Rua Paisandú,358. Cep: 99010100 - Passo Fundo, Rio Grande do Sul/RS. Brasil. Fone: +55 (59) 9 91861467. E-mail: manu.lando@hotmail.com

Research Ethics Committee Approval (if necessary): Declaramos que os pacientes aceitaram divulgação de informação presente no estudo através de termo de consentimento informado e que o estudo seguiu as diretrizes éticas estabelecidas pela Declaração de Helsinque.

Received on: Sep 7, 2021. Accepted on: Sep 17, 2021. Available online: Sep 22, 2021.

\section{Resumo}

Os Gliomas são os tumores cerebrais mais prevalentes e representam 33\% de todos os casos de tumores cerebrais já descritos, são diferenciados conforme localização, histologia, genética e agressividade. Os gliomas de alto grau possuem um potencial de agressividade elevado e prognóstico extremamente reservado, sendo as histologias raras e atípicas ainda mais agressivas e sombrias. $\mathrm{O}$ presente trabalho, visa relatar uma série de casos acerca dos tumores cerebrais mais prevalentes, designados gliomas, em especial os relacionados as apresentações de alto grau, levando em considerações a apresentação clínica, fisiopatologia, métodos diagnósticos e tratamentos relacionados ao tema abordado.

Palavras- chave: Oncologia; Glioma; Glioblastoma; Neurologia.

\section{Introdução}

Os gliomas, originados das células gliais, são os tumores cerebrais gliais mais prevalentes e representam $33 \%$ de todos os casos de tumores cerebrais, são diferenciados conforme localização, histologia, genética e 
agressividade. Sendo classificados como gliomas de alto e baixo grau conforme classificação anatômica e molecular, características de imagem e histológicas [1-3]. Os gliomas de alto grau possuem potencial de agressividade elevado e prognóstico extremamente reservado, sendo as histologias raras e atípicas ainda mais agressivas e sombrias [1-3].

Dessa forma, o presente trabalho visa relatar uma série de casos de pacientes diagnosticados com Glioma de alto grau e assistidos em um hospital público terciário da cidade de Passo Fundo, no Rio Grande do Sul, Brasil. No desenvolvimento deste trabalho, os autores realizaram considerações acerca da apresentação clínica, fisiopatologia, métodos diagnósticos e tratamentos relacionados as apresentações de alto grau, consideradas mais agressivas, de rápida progressão e evolução clínica [13].

\section{Série de Casos}

\section{Caso 1}

Paciente, sexo masculino, 58 anos, procurou atendimento médico devido quadro de cefaleia iniciado há 15 dias, associado a dificuldade em desempenho acadêmico um mês antes do início do quadro relatado. Ao exame físico neurológico, não foi evidenciado déficits focais.

Foi realizado exame de Ressonância Magnética (RM) de encéfalo, a qual apresentou lesão expansiva heterogênea com epicentro no lobo temporal esquerdo, com hipersinal T2 e hipossinal T1, sem restrição a difusão, apresentando realce heterogêneo e periférico irregular pelo agente paramagnético, delimitando área central cística/necrótica e com alguns focos de hipossinal T2/GRE de permeio, com calcificações e medindo cerca de 5,4 $x 3,4 \mathrm{~cm}$ nos maiores eixos axiais (Figura 1).

Durante transoperatório, o paciente apresentou quadro de sangramento importante. Após cirurgia, foi realizada Tomografia Computadorizada (TC) de controle na qual evidenciou hemoventrículo, sendo necessário realizar Derivação Ventricular Externa (DVE). O paciente apresentou quadro de meningite como complicação pós-operatória, recebendo alta hospitalar após 28 dias de antibioticoterapia baseada em Meropenem e Vancomicina, apresentando hemiparesia esquerda leve.

O exame anatomopatológico foi compatível com glioma de alto grau e a imuno-histoquímica, com gliosarcoma, apresentando GFAP e OLG2 focalmente positivos, Ki-67: $60 \%$, e ATRX positivo. Todavia, houve progressão de doença evidenciada por recidiva de lesão, quinze dias após alta hospitalar, manifestada com piora do nível de consciência. Desta forma, paciente e 


familiares optaram em seguir apenas com cuidados paliativos.

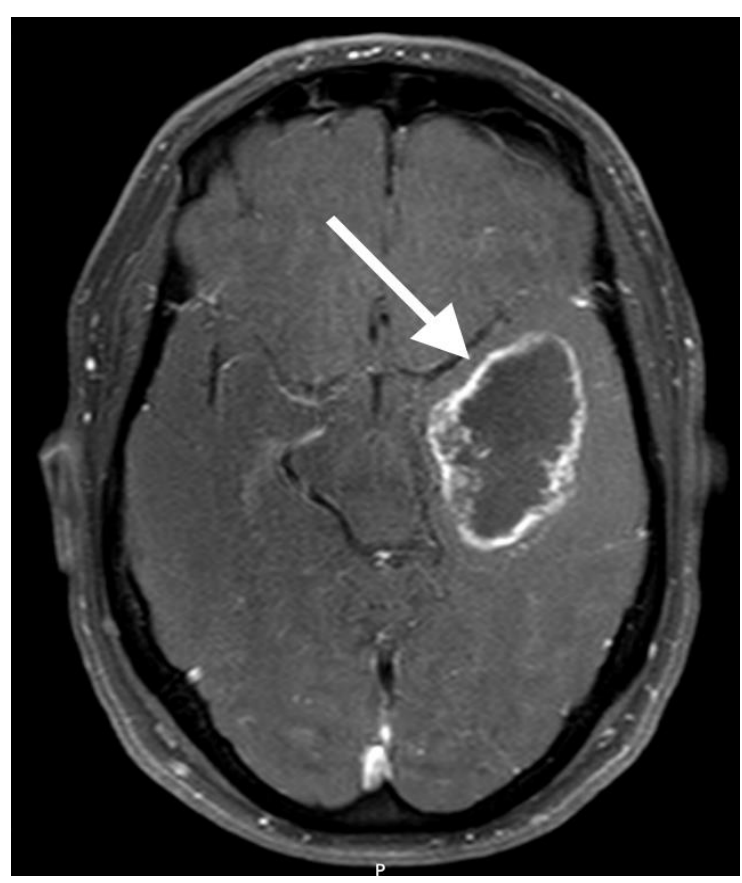

Figura 1: Imagem de ressonância magnética no plano axial, ponderada em T1 e após injeção de contraste paramagnético, demonstrando massa (seta branca) na região núcleo-capsular esquerda, com realce periférico, determinando efeito de massa sobre o hipocampo e o pedúnculo cerebral esquerdos.

\section{Caso 2}

Paciente masculino, 65 anos, encaminhado ao serviço de neurocirurgia por apresentar alterações comportamentais há 15 dias, com piora no dia da internação. No exame físico de admissão, apresentava quadro clínico de hemiparesia à esquerda associada a paralisia facial central e à esquerda. Foi realizada RM de encéfalo, na qual evidenciou lesão expansiva e infiltrativa em região insular e temporal do hemisfério cerebral esquerdo, com sinal intermediário em T2/Flair, hipossinal T1 e restrição a difusão, apresentando realce heterogêneo e periférico pelo agente paramagnético, delimitando áreas císticas/necróticas de permeio, medindo cerca de 6,2 x 5,6 cm nos maiores eixos (Figura 2).

Assim, o paciente foi submetido a ressecção completa da lesão, com neuromonitorização intraoperatória. O paciente evoluiu sem complicações no pós-operatório imediato, recebendo alta hospitalar quatro dias após cirurgia.

O exame anatomopatológico da lesão foi compatível com glioma de alto grau. A análises imunohistoquímicas evidenciaram glioma de alto grau epitelióide, com Ki-67: 10\%, GFAP, OLIG2 e TRX positivos.

Como abordagem terapêutica, foi instituído tratamento adjuvante, quimioterapia baseada em 
Temozolamida (TMZ) e radioterapia. Todavia, o paciente apresentou recidiva da lesão quatro meses após o procedimento cirúrgico, sendo optado por não realizar nova ressecção, devido à topografia da lesão. Atualmente, paciente segue em acompanhamento baseado em terapia de suporte.

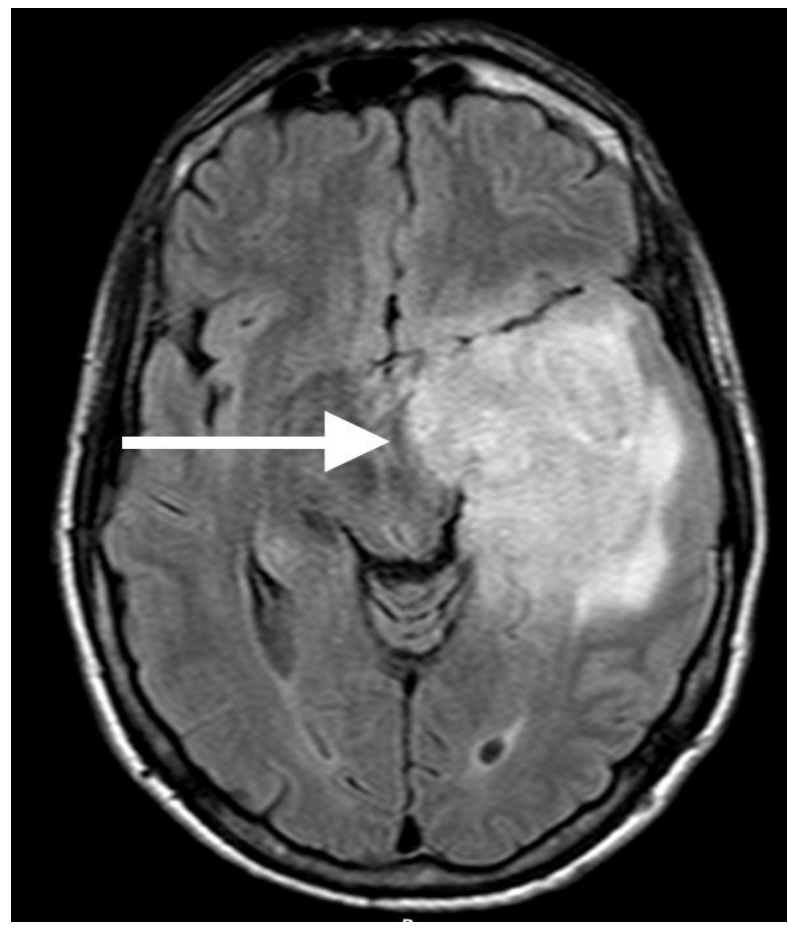

Figura 2: Imagem de ressonância magnética no plano axial, ponderada em FLAIR, demonstrando massa (seta branca) na região núcleo capsular esquerda, determinando efeito de massa sobre o lobo temporal, o hipocampo e o pedúnculo cerebral esquerdos.

\section{Caso 3}

Paciente masculino, 34 anos, iniciou quadro de zumbido associado a vertigem aproximadamente um mês antes de atendimento neurocirúrgico. Ao exame de RM de encéfalo, foi evidenciada lesão infiltrativa frontoparietal direita, com comprometimento do tálamo e extensão contralateral, através do esplênio do corpo caloso, além de infiltração do lobo temporal contralateral (Figura 3).

$$
\text { Foi realizada biópsia }
$$

estereotáxica da lesão, sendo que exame anatomopatológico foi compatível com glioma de alto grau. $\mathrm{O}$ exame de imunohistoquímica evidenciou IDH mutado e MGMT metilada.

$$
\text { Foi instituido terapia }
$$
radioterápica, 60 Gy em 30 frações, sendo finalizado todos os ciclos. Atualmente, paciente segue em acompanhamento oncológico segundo regime de tratamento quimioterápico adjuvante baseado em TMZ $200 \mathrm{mg} / \mathrm{m}^{2}$. Todavia, segundo exames de reestadiamento, paciente vem apresentando progressão do quadro oncológico. 


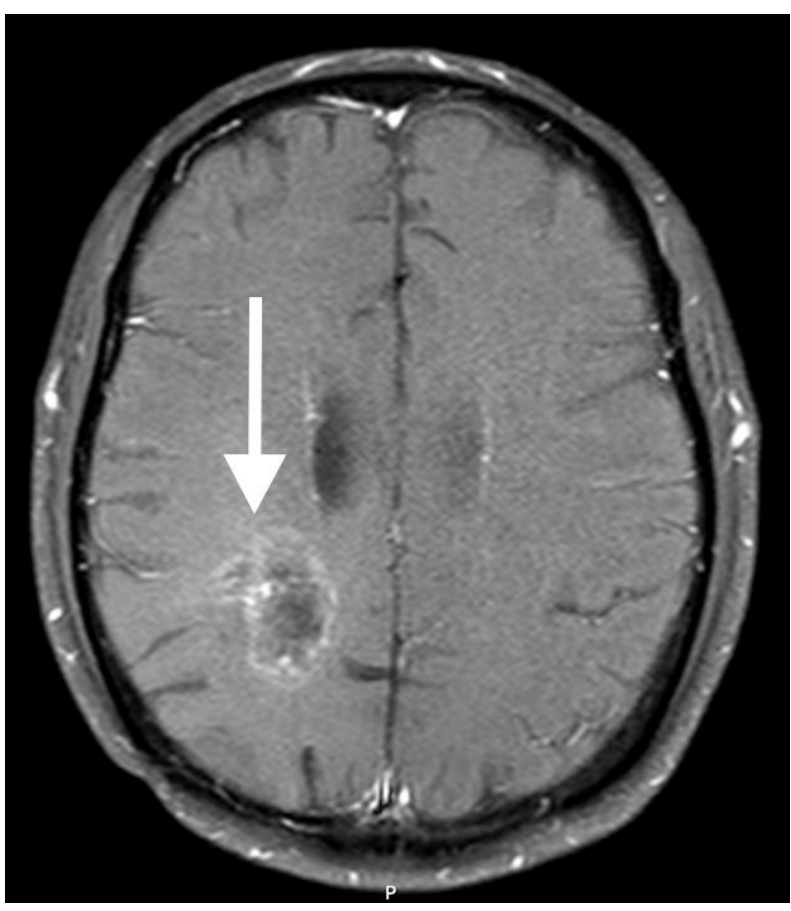

Figura 3: Imagem de ressonância magnética no plano axial, ponderada em T1 e após injeção de contraste paramagnético, demonstrando massa (seta branca) na substância branca do lobo parietal direito, com realce periférico.

\section{Caso 4}

Paciente masculino, 63 anos, previamente hígido, iniciou quadro de vertigem associado a déficit de memória anterógrada. A partir de investigação clínica com neuro-imagem, foi evidenciadalesão frontal à esquerda com invasão do corno frontal do ventrículo lateral ipsilateral (Figura 4).

O paciente foi encaminhado para cirurgia de resseção de lesão. Entretanto, de modo emergencial, o paciente foi submetido a craniotomia descompressiva devido ao edema cerebral.

O paciente evoluiu seu pós operatório com quadro clínico de hidrocefalia e DVE. Posteriormente, paciente evoluiu com melhora clínica, apresentando recuperação neurológica gradual.

O exame anatomopatológico foi compatível com gliosarcoma, imunohistoquímica evidenciou GFAP e OLIG 2 focalmente positivos, Ki-67: 40\% e ATRX positivo. Dessa forma, foi realizada terapia adjuvante concomitante radioterápica, 46 Gy em 23 frações, associada a quimioterapia baseada em TMZ $75 \mathrm{mg} / \mathrm{m}^{2}$. Todavia, visto a evolução com piora gradual do quadro apresentado, foi instituído terapia suporte, sendo que o paciente veio a óbito em 6 meses após o procedimento cirúrgico realizado.

Todos os pacientes relatados foram acompanhados desde o início do diagnóstico até o desfecho no qual ocorreu falecimento ou foi optado por cuidados paliativos exclusivos. Todavia 
aqueles que não apresentaram tal seguimento clínico oncológico. desfecho continuam em tratamento/

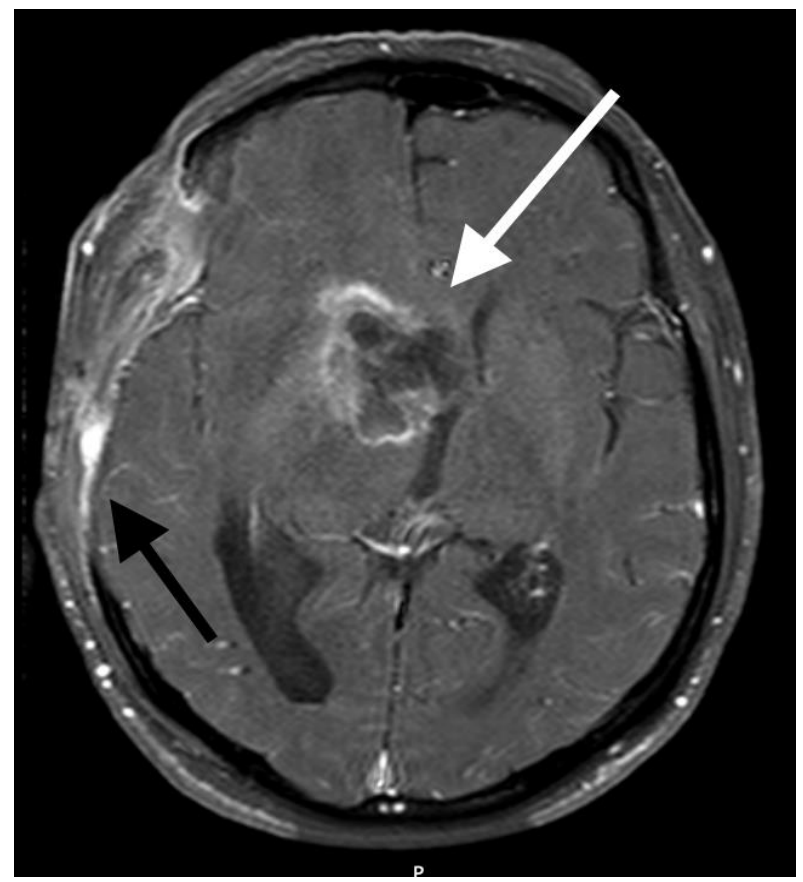

Figura 4: Imagem de ressonância magnética no plano axial, ponderada em T1 e após injeção de contraste paramagnético, demonstrando massa (seta branca) na região núcleo-capsular direita, com realce periférico, determinando efeito de massa com desvio das estruturas da linha média para a direita. Observam-se também alterações cirúrgicas de craniectomia (seta preta).

\section{Discussão e Conclusão}

O Glioblastoma Multiforme (GBM) é a neoplasia cerebral primaria mais comum e altamente agressiva, pertencendo a classificação de glioma grau IV da OMS. O Gliosarcoma (GS), trata-se de um raro subtipo tumoral, variante do Isocitrato Desidrogenase (IDH) tipo selvagem, cerca de $2 \%$ de todos GBM, cuja prevalência diagnóstica é superior em pacientes masculinos em faixa etária adulta [4].
O Gliosarcoma apresenta prevalência superior em região supratentorial, composto por padrão histológico associado a um crescimento bifásico, com células gliais associadas a elementos sarcomatosos [5-7]. Sendo associado a padrão metastático extracraniano em cerca de $11 \%$ de suas apresentações [8-9]. Padrão o qual diferencia o mesmo do GBM, qual apresenta prevalência inferior a $2 \%$ relacionada a tal processo metastático [10-11]. 
A despeito do glioblastoma epiteliide, um raro subtipo variante do GBM, este é responsável por cerca de $1,7 \%$ do total dos casos, sendo caracterizado por alto potencial de agressividade e prevalência diagnóstica predominante por pacientes em faixa etária infantil e adultos jovens [11-12].

A localização relacionada a apresentação do glioblastoma epitelioide geralmente é em regiões hemisféricas cerebrais, além de região de diencéfalo [11-14]. Cuja apresentação é composta por mutações em gene BRAF-V600E, presentes em até 53,8\% de suas manifestações, além de positividade para marcadores $\mathrm{S} 100$ e VIM [13-14]. Sendo esse subtipo tumoral, associado a uma sobrevida diagnóstica relativamente curta, cerca de 5 a 6 meses, em relação aos demais glioblastomas, o que comprova a rápida progressão de doença associada ao elevado padrão agressivo apresentado [13-14].

A cerca de métodos diagnósticos, em relação aos gliomas, o histórico clínico, o exame físico associado ao neurológico são de extrema relevância, bem como exames de imagem complementares. Tendo em vista que a RNM auxilia na acurácia diagnóstica, uma vez que tal método é capaz de detectar cerca de 95\% dos tumores cerebrais e a origem dos mesmos [11$14]$.
A confirmação diagnóstica é realizada através de análise de biópsia da região associada a atividade tumoral, sendo assim e conforme achados imunohistoquímicos, uma decisão em relação a possível tratamento é instituída $[3,15]$.

Destaca-se no tratamento dos gliomas de alto grau, o método baseado em excisão cirúrgica, em especial a radiocirurgia esteriotáxica, seguida de quimioterapia e radioterapia adjuvantes, os quais auxiliam na melhora da função neurológica e no ganho sobrevida global [4- 5, 15].

Todavia, quando presentes mutação IGH1 e metilação do MGMT, tais achados moleculares corroboram para um pior prognóstico da doença, como foi possível evidenciar nos presentes casos relatados. Quadros de astrocitomas anaplásicos, GBM e GS, aradioterapia adjuvante com dose tumoral completa (60 Gy durante 6 semanas) deve ser considerada, bem como quimioterapia com temozolomida (TMZ) [4- 5, 15-16], as quais também foram instituídas nos pacientes relatados conforme indicação. No que tange respeito aos gliomas difusos de linha média, mesmo em cenário paliativo, a radioterapia auxilia na diminuição de progressão da doença [15-16].

Em relação ao prognóstico, a sobrevida está associada ao subtipo tumoral, histologia, localização, número 
de lesões associado ao tratamento instituído [13-18]. Os Astrocitomaas anaplásicos e o GBM variam de 50\% em um ano a 15\% em cinco anos com terapia multimodal, 30 meses e 15 meses de sobrevida média em relação ao tratamento padrão a cerca desses subtipos tumorais respectivamente [1318].

Os casos de GBM raros, como os relatados, a média de sobrevida global relacionada varia de 4 a 11,5 meses relacionado a quadros de gliosarcomas e 5 a 6 meses relacionado a quadros de glioblastoma epitelioide. No caso de gliomas difusos de linha média, a sobrevida geralmente é inferior a um ano, mesmo em casos com adoção de radioterapia paliativa associada [13-18].

Sendo assim, o presente estudo acerca das apresentações clínicas dos gliomas de alto grau, impacta positivamente para disseminação do conteúdo teórico- científico, médico/ oncológico. Através da descrição da série de caso e discussão apresentadas, foi possível elucidar a apresentação clínica, fisiopatologia, os métodos diagnósticos e os tratamentos relacionados as apresentações mais agressivas do tema abordado, ainda reservado e de pior prognóstico quando diagnosticado.

\section{Referências}

[1] Louis DN, Perry A, Reifenberger G. The 2016 World Health Organization classification of tumors of the central nervous system: A summary. Acta Neuropathol, 131 (6):803-820, 2016. doi: 10.1007/s00401-016-1545-1.

[2] Schneider T, Mawrin C, Scherlach C. Gliomas in Adults. Deutsches Aerzteblatt Online. 107 (45): 799-808, 2010. doi:10.3238/arztebl.2010.0799.

[3] Zülch KJ. Berlin-Heidelberg-New York: Springer Verlag. Brain Tumors. Their biology and pathology. 1986.

[4] Han S.J, Yang I, Tihan, T. Primary gliosarcoma: key clinical and pathologic distinctions from glioblastoma with implications as a unique oncologic entity. Journal of Neuro-Oncology, 96 (3): 313-320, 2009. doi:10.1007/s11060009-9973-6.

[5] Morantz R.A, Feigin I, Ransohoff. J.Clinical and pathological study of 24 cases of gliosarcoma. Journal of Neurosurgery, 45 (4): 398-408, 1976. doi:10.3171/jns.1976.45.4.0398.

[6] Pareckh H.C, O'Donovan D.G, Sharma R.R. Report of 17 cases. British Journal of Neurosurgery, 9 (2):171-178, 1995. doi:10.1080/02688699550041511.

[7] Peckham M. E, Osborn A. G, Palmer C. A. Gliosarcoma: Neuroimaging and Immunohistochemical Findings. Journal of Neuroimaging, 29: 126-32, 2018. doi:10.1111/jon.12565.

[8] Beaumont T. L, Kupsky W. J, Barger G. R. Gliosarcoma with multiple 
extracranial metastases: case report and review of the literature. Journal of Neuro-Oncology, 83 (1): 39-46, 2007. doi:10.1007/ s11060-006-9295-x.

[9] Piccirilli M, Brunetto G. M. F, Rocchi G. Extra Central Nervous System Metastases from Cerebral Glioblastoma Multiforme in Elderly Patients. ClinicoPathological Remarks on our Series of Seven Cases and Critical Review of the Literature. Tumori Journal, 94 (1): 40-51, 2008. doi:10.1177/0 30089160809400109.

[10] Pietschmann S, Von Bueren, A. O, Henke G. An individual patient data meta-analysis on characteristics, treatments and outcomes of the glioblastoma/gliosarcoma patients with central nervous system metastases reported in literature until 2013. Journal of Neuro-Oncology, 120 (3): 451-457, 2014. doi:10.1007/s11060-014-1596-x.

[11] Louis D.N, Ohgaki H, Wiestler O. D. The 2007 WHO Classification of Tumours of the Central Nervous System. Acta Neuropathologica, 114 (2): 97-109, 2007. doi:10.1007/s00401-0070243-4.

[12] Broniscer A, Tatevossian R. G, Sabin N. D. Clinical, radiological, histological and molecular characteristics of paediatric epithelioid glioblastoma. Neuropathology and Applied Neurobiology, 40 (3): 327-336, 2014. doi:10.1111/nan.12093.

[12] Kleinschmidt-DeMasters, B. K, Aisner D L, Birks D. K. Epithelioid GBMs Show a High Percentage of BRF V600E Mutation. The American Journal of Surgical Pathology, 37 (5): 685-698, 2013. doi:10.1097/pas.0b013e31827f9c5.

[13] Ellison DW, Kleinschmidt- De Masters BK, Park SH. Epithelioid Glioblastoma. In WHO Classification of Tumours of the Central Nervous System. 4th Revised Ed.

[14] Louis DN, et al editors. International Agency for Research on Cancer, Lyon, 2016. pp 50-1.

[15] Stupp R, Taillibert S, Kanner A. Effect of tumor-treating fields plus maintenance temozolomide vs maintenance temozolomide alone on survival in patients with glioblastoma: A randomized clinical trial. JAMA 318 (23):2306-2316, 2017. doi: 10.1001/ jama.2017.18718.

[16] Buckner JC, Shaw EG, Pugh SL. Radiation plus procarbazine, CCNU, and vincristine in lowgrade glioma. $\mathrm{N}$ Engl J Med 374 (14):1344-1355, 2016. doi: 10.1056/NEJMoa1500925.

[17] Courville CB. Multiple primary tumors of the brain: review of the literature and report of twenty one cases. Am J Cancer, 26:703-731, 1936.

[18] Choucair AK, Levin VA, Gutin PH. Development of multiple lesions during radiation therapy and chemotherapy in patients with gliomas. J Neurosurg, 65:654-658, 1986. doi: 10.3171/ jns.1986.65.5.0654.

Conflito de interesse: Os autores declaram não haver conflitos de interesse.

Agradecimentos: Agradecemos a toda equipe pelo excelente manejo 
multidisciplinar e especialmente ao Hospital de Clínicas de Passo Fundo na contribuição e fornecimento de dados para realização do presente trabalho.

Financiamento: Não se aplica.

How to cite this article: Pastorello J, Lando E, Amaral CS, Cechetti CP, Vacaro GZ, Henn NT, Bueno MR, Cogo DC, Rottenfusser R, Mesquita Filho PM. Série de Casos: Gliomas de Alto Grau do Hospital de Clínicas de Passo Fundo HCPF - RS. Brazilian Journal of Case Reports. 2021Jul-Sep;01(3):162-171. 